\title{
A particular anatomic variation of the course of the left pulmonary artery
}

Giulio Melloni, MD, a George Cremona, MD, ${ }^{\text {b }}$ Angelo Carretta, MD, ${ }^{a}$ Luca Ferla, MD, ${ }^{a}$ Alessandro Bandiera, MD, ${ }^{\text {a }}$ and Piero Zannini, MD, ${ }^{\text {a }}$ Milan, Italy

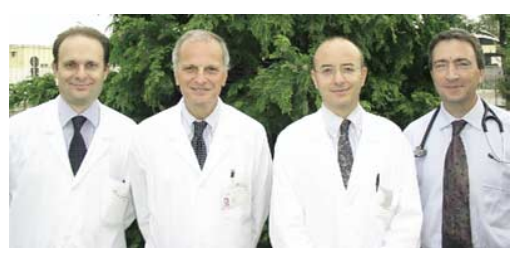

$\mathrm{K}$ nowledge of the anomalies of the anatomic course of the pulmonary artery is important for the thoracic surgeon. Some of these variations are extremely frequent, whereas some others are exceptional. Detailed knowledge of these anomalies avoids inadvertent vascular lesions potentially causing life-threatening perioperative bleeding.

\section{Clinical Summary}

PATIENT 1. A 61-year-old man underwent left upper lobectomy for T2 N0 squamous cell carcinoma. During the operation, an anomalous left pulmonary artery course was noted. The left pulmonary artery passed into the bifurcation between the common bronchus to the anterior and apical posterior segments and the lingular bronchus (Figure 1). The vascular anomaly prevented adequate exposure of the origin of the left upper bronchus. Sepa-

From the Department of Thoracic Surgery a and the Pulmonary Medicine Unit, ${ }^{\text {b }}$ Scientific Institute H San Raffaele, Milan, Italy.

Received for publication July 1, 2005; accepted for publication Aug 4, 2005.

Address for reprints: Giulio Melloni, MD, Unità Operativa di Chirurgia Toracica, Ospedale San Raffaele, Via Olgettina, 60, 20132, Milan, Italy (E-mail: giulio.melloni@hsr.it).

J Thorac Cardiovasc Surg 2005;130:1719-20

$0022-5223 / \$ 30.00$

Copyright $\odot 2005$ by The American Association for Thoracic Surgery doi:10.1016/j.jtcvs.2005.08.009 rate manual closure of the common bronchus to the anterior and apical posterior segments and the lingular bronchus was therefore performed. The proximity of the bronchial stumps to the pulmonary artery made it necessary to interpose a pericardial fat pad between the airway and the artery.

PATIENT 2. A 56-year-old man underwent left upper lobectomy for T2 N0 adenocarcinoma. An identical anomalous left pulmonary artery course was discovered, necessitating separate closure of the 2 bronchi with the interposition of a pericardial fat flap between the bronchial stumps and the artery.

\section{Discussion}

This type of anomaly of the left pulmonary artery course is exceptional and not described in standard textbooks of thoracic surgery. To the best of our knowledge, only one case report has been published in the recent international literature. ${ }^{1}$ However, we experienced this anatomic variation in 2 of 125 patients undergoing left upper lobectomies in the last 5 years. This relatively high incidence (1.6\% of all left upper lobectomies) seems therefore to contradict the reported rarity of this anatomic variation.

In the only case reported in the literature, ${ }^{1}$ the vascular variation was associated with a bronchial anomaly. In this patient a left upper lobe bronchial trunk and a distinct lingular bronchial trunk arising from the anterior aspect of a very short left bronchus intermedius were present, mimicking the right bronchial anatomy. There was no associated bronchial anomaly in our 2 patients, and
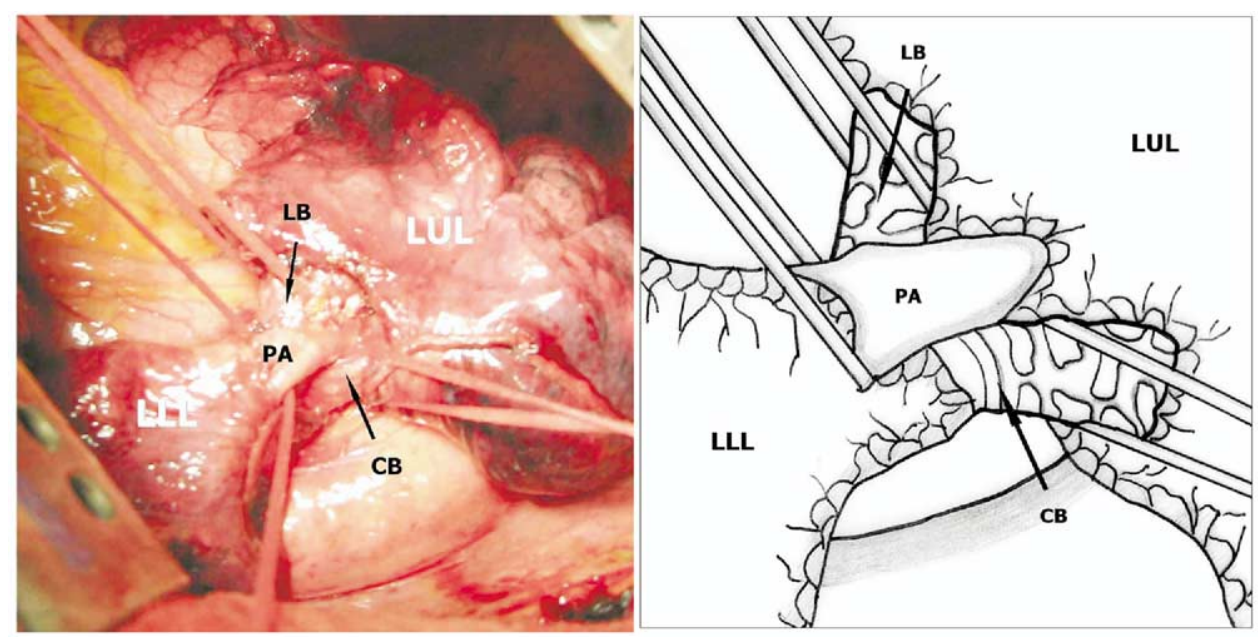

Figure 1. A, Operative view; B, schematic representation. LUL, Left upper lobe; $L L L$, left lower lobe; $P A$, pulmonary artery; $C B$, common bronchus to the anterior and apical posterior segments; $L B$, lingular bronchus. 


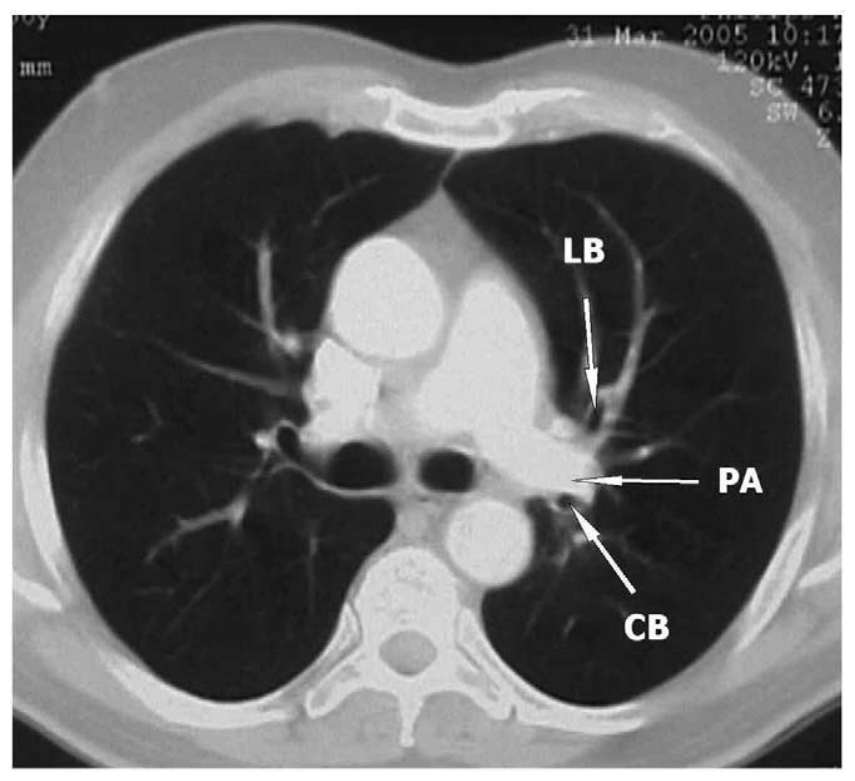

Figure 2. Preoperative computed tomographic scan of the chest showing the anomaly of the course of the left pulmonary artery. $P A$, Pulmonary artery; $C B$, common bronchus to the anterior and apical posterior segments; $L B$, lingular bronchus. this was confirmed by the preoperative bronchoscopy, which showed normal anatomy in both cases.

In our 2 patients the anomaly of the course of the left pulmonary artery precluded adequate dissection of the origin of the left upper bronchus and mandated separate closure of the common bronchus to the anterior and apical posterior segments and the lingular bronchus. In this particular anatomic situation, the interposition of neighboring tissue, such as pericardial fat, is a precautionary measure to prevent postoperative bronchovascular fistulas.

This anatomic variation was not identified preoperatively in either of the 2 patients. However, on review of the computed tomographic scan, the arterial anomaly could be clearly identified (Figure 2).

In conclusion, the particular anatomic variation of the left pulmonary artery described in this article, unassociated with bronchial anomalies, seems to demonstrate a relatively high frequency. Detailed knowledge of this vascular anomaly and its potential presence is therefore important for the training and practicing thoracic surgeon.

\section{Reference}

1. Galetta D, Debrosse D, Gossot D, Serra M, Grunenwald D. Anomalous left bronchial segmentation associated with an abnormal left pulmonary artery course. Ann Thorac Surg. 2004;78:e16.

\section{A modified elephant trunk technique: The 3-fold elephant trunk technique}

Kenji Okada, MD, Taijiro Sueda, MD, Kazumasa Orihashi, MD, Katsuhiko Imai, MD, and Katsutoshi Sato, MD, Hiroshima, Japan

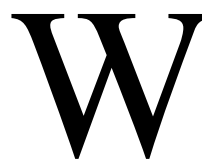

e report a modified elephant trunk (ET) technique, the 3-fold ET technique. This technique is an alternative for preventing thromboembolic events and paraplegia.

\section{Technical Description}

The reversed and bidirectional $\mathrm{ET}^{1}$ is made by invaginating the prosthesis. The idea for this modified technique arose from envelop-

\footnotetext{
From the Department of Cardiovascular Surgery, Hiroshima University Hospital, Hiroshima, Japan.

Received for publication July 6, 2005; accepted for publication Aug 3, 2005.

Address for reprints: Kenji Okada, MD, Department of Cardiovascular Surgery, Hiroshima University Hospital, 1-2-3 Kasumi, Minami-ku, Hiroshima, 734-8551, Japan (E-mail: kokada@ hiroshima-u.ac.jp).

J Thorac Cardiovasc Surg 2005;130:1720-2

$0022-5223 / \$ 30.00$

Copyright $\odot 2005$ by The American Association for Thoracic Surgery doi:10.1016/j.jtcvs.2005.08.011
}

ing the prosthesis instead of invaginating it. A single and straight prosthesis is folded twice and made into a 3 -fold ET (Figure 1). The first fold is placed about $7 \mathrm{~cm}$ from the end. The second fold is made backward, $5 \mathrm{~cm}$ back to the proximal site. In other words, the distance between the proximal edge and the second fold is about $2 \mathrm{~cm}$. Two stitches are placed on each of the proximal and distal sides to fix the ET in place. The stitches are put in to prevent the invasion of blood flow into the fold. The ET is about $7 \mathrm{~cm}$ in length folded and $17 \mathrm{~cm}$ in length unfolded.

During the first operation, the prefabricated prosthesis is inserted into the distal aneurysm while the ascending aorta and aortic arch are repaired. During the second operation, a clamp is put on a $2-\mathrm{cm}$ single tube at the proximal side, and then the aneurysm is opened. At the proximal site, the ET is located within the lumen. The 4 stitches are pulled away, and the ET is pulled out. Then the prosthesis is pulled open. The triple prosthesis with 2 folds is stretched as an unfolded, straight, and single graft. Then the residual aneurysm is opened, and a proximal anastomosis is made between the 3-fold ET and the new prosthesis. 